IEEE Particle Accelerator Conference, $J / .</ y /-3 / .0 /=/$, Vancouver, Canada*

\title{
A High Resolution Electron Beam Profile Monitor
}

\author{
W.S. Graves, E.D. Johnson \\ NSLS, Brookhaven National Laboratory, Upton, NY 11973 USA \\ P.G. O' Shea, Duke University, Durham, NC USA
}

CONF- 97050

\section{Abstract}

A new beam diagnostic to measure transverse profiles of electron beams is described. This profile monitor uses a Yttrium:Aluminum:Garnet (YAG) crystal doped with a visible-light scintillator to produce an image of the tranverse beam distribution. The advantage of this material over traditional fluorescent screens is that it is formed from a single crystal, and therefore has improved spatial resolution. The current system is limited to a resolution of about 10 microns. Improvements in the optical transport will enable measurements of RMS beam sizes of less than $1 \mathrm{mi}-$ cron. The total cost of the system is modest.

\section{INTRODUCTION}

Fluorescent screens are widely used to measure the transverse profiles of electron beams at high-energy accelerator facilities. Traditional fuorescent screens[1], such as ZnS, have an individual grain size of $50-100$ microns. This limits the image resolution to the same scale. The high brightness electron beams now being produced for short wavelength FELs and high energy colliders have focused sizes of a few microns in diameter. Resolution below this is necessary for accurate reproduction of the transverse profiles. We have tested a profile monitor that meets this requirement by employing a single crystal scintillator. The material is doped so that it fluoresces in the visible. The crystal is uniform at the molecular level, which provides very high resolution by removing individual grain size as the limiting factor in measuring very small beams.

\section{MEASUREMENTS}

Measurements of electron beam sizes and of the crystal's properties were performed at both the Accelerator Test Facility (ATF) [2] at BNL and at Duke University's Free Electron Laser Laboratory [3]. At Duke the brightness, linearity, damage resistance, and time structure of the fluorescence were made while the measurements at the ATF were devoted to spatial resolution limits. The actual configurations of the two experiments differ somewhat, but they share several common elements, most notably the scintillator crystals.

The YAG crystals are supplied as disks $10 \mathrm{~mm}$ in diameter and $0.5 \mathrm{~mm}$ thick. We were concerned that these nominally insulating materials might develop potentially damaging electrostatic charge on their surfaces in the electron beam. To address this issue we used a standard electron microscopy sample preparation technique and evaporated an approximately $14 \mathrm{~nm}$ thick coating of $60 \% \mathrm{Au} / 40 \% \mathrm{Pd}$ on

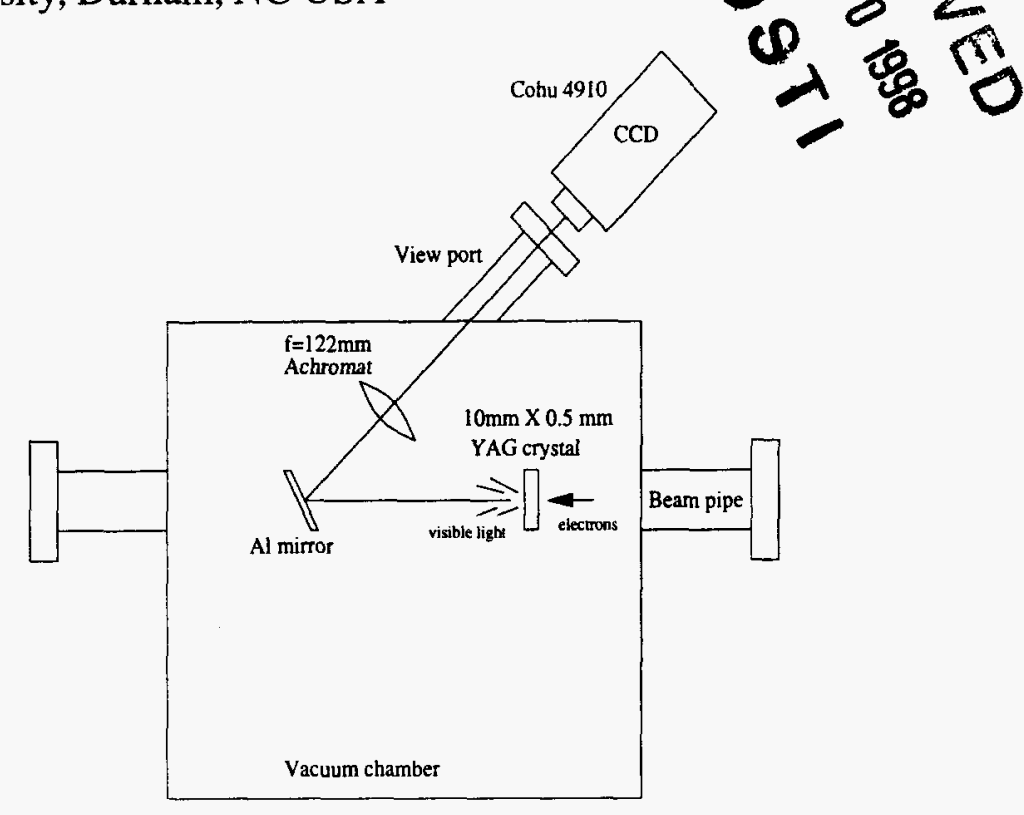

Figure 1: Optical layout of profile monitor at ATF.

both faces of the crystal. We have not run any experiments with uncoated crystals.

For these experiments the duration of the optical pulse is less than $100 \mathrm{~ns}$, therefore a camera that simultaneously exposes both interlaced video fields is necessary. For this reason, each measurement was recorded with a Cohu 4910 video camera. To provide the best image fidelity, the monitors are all arranged with the crystal at normal incidence to the electron beam. An aluminum mirror is placed downstream of the crystal to deflect the fluorescence light out to the camera. We made preliminary measurements on airports at the Duke FEL linac at 36 and $264 \mathrm{MeV}$ to make sure that the crystals were robust, and then made in-vacuum measurements at the high energy port $(264 \mathrm{MeV})$. In this configuration, the light from the crystal is brought out at 90 degrees from the electron beam, deviated and inverted by a rooftop (Amici) prism, and focused to the CCD camera by a $55 \mathrm{~mm}$ focal length telecentric lens with a $2 X$ adapter. In this arrangement the field of view of the screen was 11.6 by $9.46 \mathrm{~mm}(\mathrm{~h} \times \mathrm{v})$ corresponding to a pixel resolution of 15.5 by 16.3 microns.

To test damage resistance the YAG was inserted for an extended period in the beam, and its performance monitored. It was then removed and examined for surface damage. After 2 hours and approximately $1200 \mathrm{nC}$ of charge, no loss of intensity was observed, and no visible damage was apparent on the crystal. A PMT was used to measure the time duration of the YAG flourescence. The pulse du- 


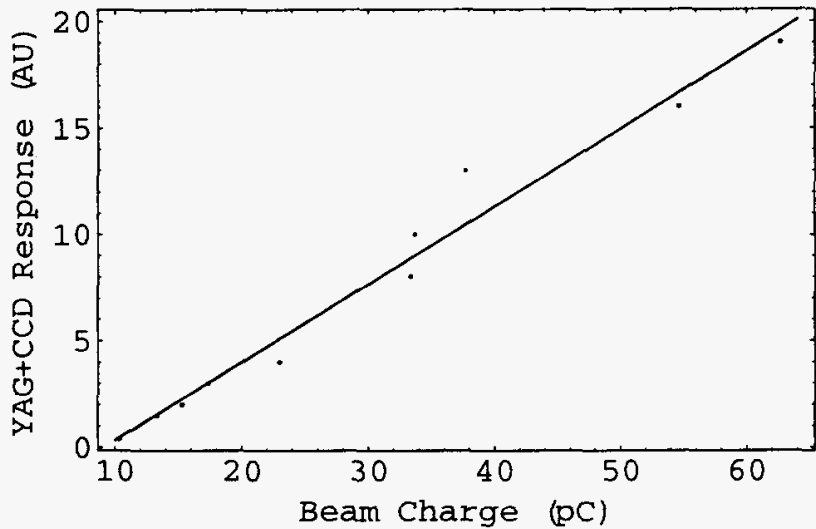

Figure 2: Linearity of YAG/CCD response. Charge variation estimated at $10 \%$ at each point.

ration was measured to be about 80 ns FWHM. Figure 2 shows a plot of light energy recorded by the CCD vs beam charge. The crystal exhibits good linearity for this range of charge. The brightness of the spot produced on the YAG was compared to similar beam spots on a $\mathrm{ZnS}$ screen. The intensity of the YAG matched that of the $\mathrm{ZnS}$.

The smallest spot sizes were produced by the high brightness electron beam at the ATF. Figure 1 shows the layout of the optical system used to measure the small beams. The system is installed as part of the Smith-Purcell experiment [4] located on beamline 1 . In this case the available ports on the vacuum chamber dictated that the optical beam be taken out at an angle of 45 degrees with respect to the electron beam axis. To improve the resolution of the experiment we also removed the telecentric lens from the camera and substituted for it a diffraction-limited achromat of $122 \mathrm{~mm}$ focal length located inside the vacuum chamber. It is positioned to produce a 1 to 1 image of the YAG outside the chamber on the CCD camera. The optical focus and magnification were calibrated by placing a metal plug with 100 micron hole at the YAG position and recording the image. After the components are locked in place, no adjustments are available. In this case, the field of view is equal to the CCD chip size, $6.4 \mathrm{~mm} \mathrm{X} 4.8 \mathrm{~mm}$ corresponding to 8.3 by 8.6 micron pixels.

The minimum electron beam size measured had a RMS radius of $10.7 \mu \mathrm{m}$ at $50 \mathrm{MeV}$ and approximately $100 \mathrm{pC}$ of charge (Fig. 4). This is consistent with normalized emittance of $0.5 \pi \mathrm{mm}-\mathrm{mrad}$ and a beta function of $2 \mathrm{~cm}$. These values are in reasonable agreement with TRANSPORT calculations.

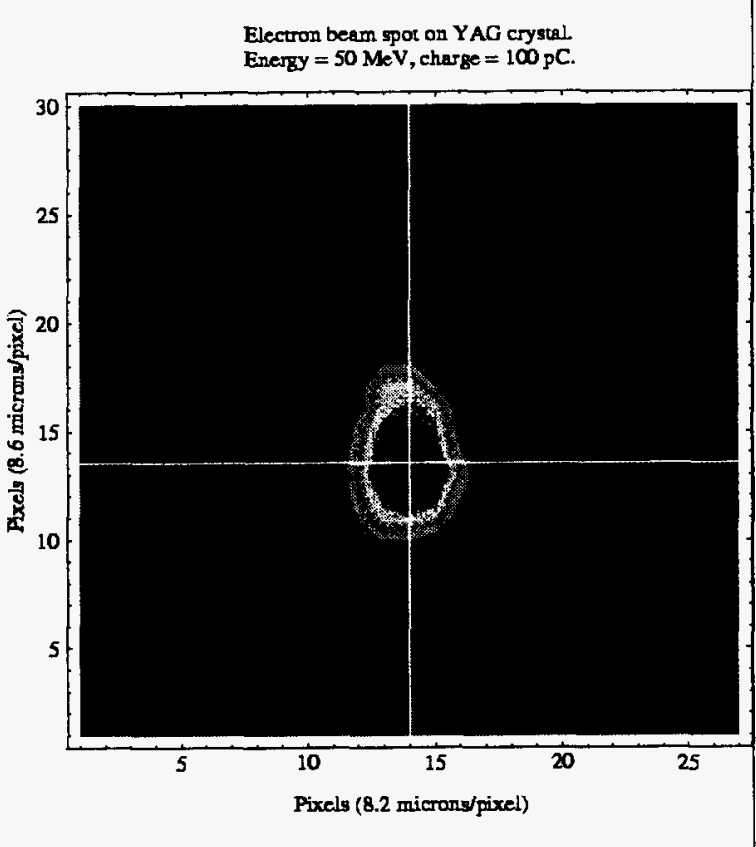

Figure 3: Smallest beam spot observed at ATF.

\section{RESOLUTION LIMITS}

The ultimate resolution is set by both the minimum object size that can be produced by the crystal and by the diffraction of visible light. The minimum object size for high energy electrons is limited by multiple Coulomb scattering of the beam through the crystal and by the generation of bremsstralung. The multiple scattering angle through the material is given approximately by [5]:

$$
\theta_{m s}(z)=\frac{13.6 \mathrm{MeV}}{p c} \sqrt{z / Z_{0}}\left[1+0.20 \ln \left(z / Z_{0}\right)\right]
$$

where $z / Z_{0}$ is the material thickness in radiation lengths and $p c$ is the electron momentum. The radiation length for YAG is $5.2 \mathrm{~cm}$. For a $50 \mathrm{MeV}$ beam traveling through a 0.5 $\mathrm{mm}$ thick crystal, $\theta_{\mathrm{ms}}=1.9 \mathrm{mrad}$, yielding a minimum possible spot size of

$$
x_{m s}=\int_{0}^{0.5 \mathrm{~mm}} \theta_{m s}(z) d z=0.6 \mu \mathrm{m}
$$

The critical energy, where energy losses due to ionization and radiation of bremsstrahlung are equal, is about 50 $\mathrm{MeV}$. Above this energy the xrays generated by electrons passing through the material will also cause scintillation, limiting the minimum measureable beam size. The characteristic emission angle of the xrays is $1 / \gamma=10 \mathrm{mrad}$ at 50 $\mathrm{MeV}$. Thus $x_{\text {brem }}=2.5 \mu \mathrm{m}$ at $50 \mathrm{MeV}$.

The minimum spot size limits due to either multiple scattering or bremsstrahlung are both dependent on the electron beam energy. The limits are reduced at higher energies (Fig. 5).

The diffraction limited diameter of a lens focus is $d_{0}=$ $2.44 \lambda(f / D)$, where the wavelength, $\lambda$, of the light is 


\section{DISCLAIMER}

This report was prepared as an account of work sponsored by an agency of the United States Government. Neither the United States Government nor any agency thereof, nor any of their employees, makes any warranty, express or implied, or assumes any legal liability or responsibility for the accuracy, completeness, or usefulness of any information, apparatus, product, or process disclosed, or represents that its use would not infringe privately owned rights. Reference herein to any specific commercial product, process, or service by trade name, trademark, manufacturer, or otherwise does not necessarily constitute or imply its endorsement, recommendation, or favoring by the United States Government or any agency thereof. The views and opinions of authors expressed herein do not necessarily state or reflect those of the United States Government or any agency thereof. 

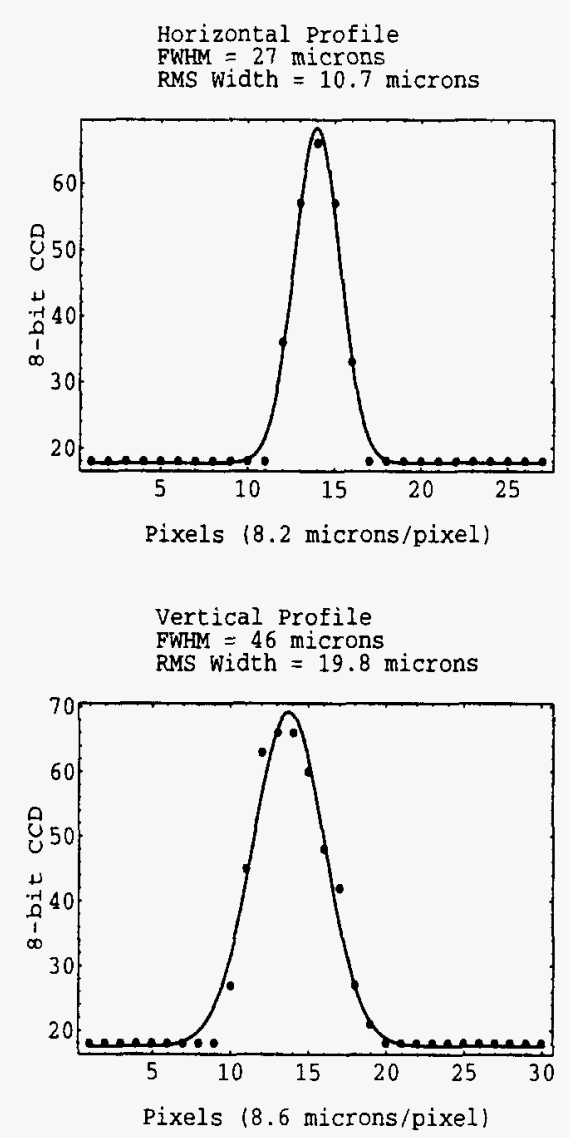

Figure 4: Smallest measured electron beam profiles.

$550 \mathrm{~nm}, f$ is the lens focal length, and $D$ is the lens diameter. This is the diameter of the zeroes of the Airy disk. For reasonable working distances of a few centimeters, the lowest diffraction-limited $f / D$ that can be achieved is about 3. Therefore the optics of visible light limit our resolution to beam spots of 4 microns diameter, or 0.7 microns RMS size. Figure 5 summarizes the RMS spot size limits due to multiple scattering, bremsstrahlung, and diffraction. The optics installed on our system have a magnification of unity and diffraction limited resolution of 6 microns. The camera pixel size is 8.5 microns. This pixel size currently sets the resolution limit.

Diffraction-limited resolution may be achieved by using two achromats to produce a 1 to 1 relay image outside of the vacuum chamber. Assuming that resolution of approximately 1 micron is required, the intermediate image may be magnified by a factor of 10 using a flat-field microscope objective. A CCD camera with 6-10 micron pixels would then provide the required resolution.

\section{CONCLUSIONS}

RMS beam sizes as small as 10.7 microns have been measured using a new flourescent screen based on a YAG crys-

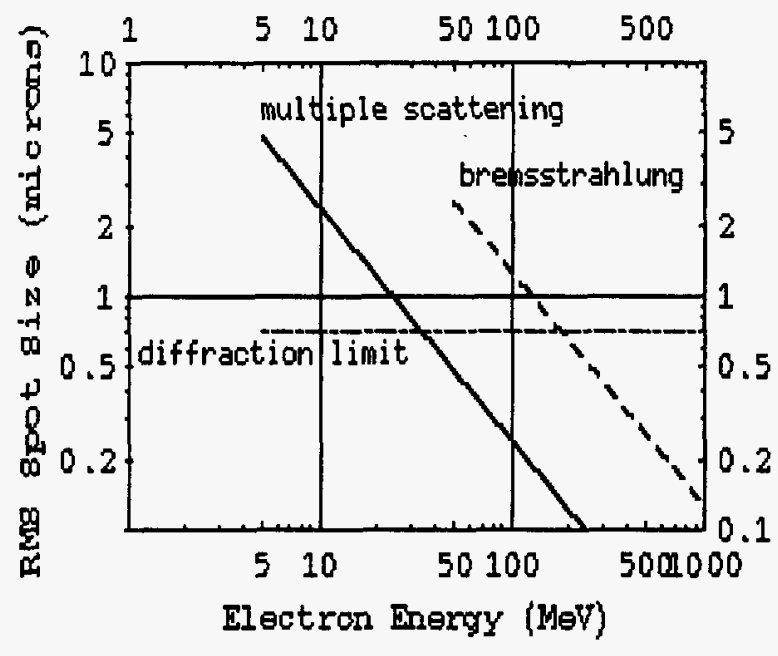

Figure 5: Effects that limit minimum spot size that may be measured as a function of electron beam energy for a 0.5 mm thick crystal.

tal. This beam size is at the limit of the current optical resolution. The resolution may be improved by using more lens elements with greater magnification. Diffraction of visible light limits the resolution to 0.7 micron RMS beam sizes. The crystal has been shown to be sensitive to small beam charges, to have a linear response, and to be damage resistant.

One of the important features of this profile monitor is its low cost. The system described here, including achromat, YAG crystal, CCD camera, and mounting hardware can be purchased for under $\$ 1500$ US.

\section{ACKNOWLEDGEMENTS}

We are grateful to the accelerator operators at the Duke FEL facility, to Vitaly Yakimenko of the ATF for producing the very small measured beams, and to Harold Kirk of the Physics Dept at BNL for assistance with installation and mounting of the profile monitor at the ATF. This work performed under the auspices of the U.S. Department of Energy Contract DE-AC02-76CH00016. The work at Duke University supported by USASDC Contract DASG-60-84C-0028.

\section{REFERENCES}

[1] J.T. Seeman, V. Luth, M. Ross, J. Sheppard. Beam tests of phosphorescent screens. Technical report, SLAC, 1985. Single Pass Collider Memo CN-290.

[2] I. Ben-Zvi et al. The Accelerator Test Facility at BNL. Nuc. Inst. and Meth., A296:348-350, 1990.

[3] P.G. O' Shea et al. Single bunch injection system for storage ring FEL using an if photoinjector. These proceedings

[4] K.Woods, J. Walsh, R. Stoner, H. Kirk, R. Fernow. The Smith Purcell experiment. Phys. Rev. Lett., 74:3808-3811, 1995.

[5] Particle Data Group, editors. Particle Properties Data Booklet. North-Holland, 1990. 
M98002593

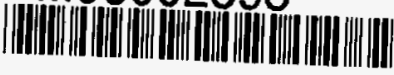

Report Number (14) BNL-65006
CONE-970503=-

subl. Date (11) 199712

sponsor code (18) DOE/ER , XF

IC Category (19) UC-400,DOE/ER

DOE 